\title{
Sam68 Modulator CWP232291
}

National Cancer Institute

\section{Source}

National Cancer Institute. Sam68 Modulator CWP232291. NCI Thesaurus. Code C97663.

A small molecule and prodrug of CWP232204 targeting Src associated in mitosis, of 68 kDa (Sam68 or KHDRBS1), with potential antineoplastic activity. CWP232291 is converted in serum into its active form CWP232204 which binds to Sam68, thereby resulting in the induction of apoptosis in selective cancer cells. Due to the multimodular structure of Sam68, the apoptosis mediated by CWP232204-Sam68 interaction can attribute from 1) activation of transcription factor NF-kB induced by tumor necrosis factor alpha signaling, 2) alternative splicing of BCL-2 apoptosis gene, driving the balance towards pro-apoptotic as opposed to anti-apoptotic isoforms, 3) down-regulation of the anti-apoptotic protein survivin via Wnt signaling. Sam68, a KH domain RNA-binding protein belonging to the signal transduction and activation of RNA (STAR) family, plays a key role in various cellular processes including cell cycle progression and apoptosis; it is upregulated in many types of cancer cells and its expression is associated with increased cell proliferation and survival. 